\title{
Iterative Computation of Polyhedral Invariants Sets for Polynomial Dynamical Systems
}

\author{
Mohamed Amin Ben Sassi *, Antoine Girard ${ }^{\dagger}$ and Sriram Sankaranarayanan* \\ * University of Colorado, Boulder, USA. \\ $\dagger$ Laboratoire Jean Kuntzmann, Université de Grenoble, France.
}

\begin{abstract}
This paper deals with the computation of polyhedral positive invariant sets for polynomial dynamical systems. A positive invariant set is a subset of the state-space such that if the initial state of the system belongs to this set, then the state of the system remains inside the set for all future time instances. In this work, we present a procedure that constructs an invariant set, iteratively, starting from an initial polyhedron that forms a "guess" at the invariant. At each iterative step, our procedure attempts to prove that the given polyhedron is a positive invariant by setting up a non-linear optimization problem for each facet of the current polyhedron. This is relaxed to a linear program through the use of the blossoming principle for polynomials. If the current iterate fails to be invariant, we attempt to use local sensitivity analysis using the primal-dual solutions of the linear program to push its faces outwards/inwards in a bid to make it invariant. Doing so, however, keeps the face normals of the iterates fixed for all steps. In this paper, we generalize the process to vary the normal vectors as well as the offsets for the individual faces. Doing so, makes the procedure completely general, but at the same time increases its complexity. Nevertheless, we demonstrate that the new approach allows our procedure to recover from a poor choice of templates initially to yield better invariants.
\end{abstract}

\section{INTRODUCTION}

This paper presents an iterative algorithm for discovering polyhedral positive invariant sets for polynomial dynamical systems. A set $P$ is a positive invariant iff for all $x \in P$, the trajectory initialized to $x(0)=x$ satisfies $x(t) \in P$ for all $t \geq 0$. Positive invariants are useful in the analysis of dynamical systems for proving properties. The problem of finding the smallest positive invariant that contains a given set (and/or excludes a set of unsafe states) is a useful primitive in reachability analysis. Likewise, the problem of finding the largest positive invariant containing an attractor is useful in bounding the region of attraction.

However, finding positive invariants is often a hard problem for nonlinear systems. Existing approaches rely on relaxations such as sum-of-squares programming to find a polynomial $p$ such that $p \geq 0$ is a positive invariant [PJP07]. In this work, we find a polyhedron that is positive invariant for a polynomial system by solving a series of linear programming (LP) problems obtained by relaxing nonlinear optimization problems. The relaxation uses the blossom principle of writing a polynomial $p$ as a multiaffine polynomial over a larger set of variables. Our overall approach can be seen as a gradient descent to discover the facets of a positive invariant polyhedron $A x \leq b$. Starting from an initial guess, each step checks using the LP relaxation, whether the current iterate $A_{j} x \leq b_{j}$ satisfies the positive invariance conditions.
Otherwise, we update our polyhedron $\left(A_{j}+U_{j}\right) x \leq b_{j}+\alpha_{j}$ by finding directions $U_{j}, \alpha_{j}$. A key insight is the use of local sensitivity analysis to find fruitful directions $U_{j}$ and $\alpha_{j}$. Our approach is implemented in MATLAB $(\mathrm{tm})$. We evaluate our approach on small but complex nonlinear systems to demonstrate the use some inital guess ( not necessarily an invariant), to iteratively derive a positive invariant.

\section{A. Related Work}

This paper generalizes the approach presented in [BG12a], where an iterative approach to the computation of polyhedral invariant sets for polynomial systems is proposed. In that work, the orientation of the facets of the polyhedra remain the same and the search for an invariant is done over the position of these facets, i.e, $U_{j}$ is always set to 0 . This is not the case for the present work. Therefore, our approach is less dependent on the initial polyhedron used. Our approach generalizes the specific case considered by Belta et al. [BH06] where a characterization of rectangular invariants for multi-affine dynamical systems is given. Other existing approaches for computing positive invariant sets for polynomial systems usually seek algebraic/semialgebraic invariants. The associated polynomial constraint solving problems are then solved using computational tools from algebraic geometry [San10] (Groebner basis), sumof-squares relaxations [PJP07], or moments of occupation measures [KHJ13]. Our approach limits the search to convex polyhedral sets and is based on linear programming and thus offers an alternative solution to the works mentioned above. Finally, this paper restricts itself to the case of autonomous systems, without controls and disturbances.Extensions for robust control invariant sets can be obtained using the method presented in our earlier work [BG12b].

\section{PRELIMINARIES}

This section presents the preliminary notions that underlie our work, including the blossoming principle. All the results in this section are quite standard, and are therefore stated without proofs. Let $R_{n}=\prod_{k=1}^{k=n}\left[a_{k}, b_{k}\right]$ be a rectangle of $\mathbb{R}^{n}$ and let $V_{n}=\prod_{k=1}^{k=n}\left\{a_{k}, b_{k}\right\}$ be its set of vertices.

\section{A. Multivariate polynomials}

A multivariate polynomial $p: \mathbb{R}^{n} \rightarrow \mathbb{R}$ is of the form

$p\left(x_{1}, \ldots, x_{n}\right): \sum_{\left(k_{1}, \ldots, k_{n}\right) \in \Delta} p_{k_{1}, \ldots, k_{n}} x_{1}^{k_{1}} \ldots x_{n}^{k_{n}}$, wherein 
$\Delta=\left\{0, \ldots, \delta_{1}\right\} \times \cdots \times\left\{0, \ldots, \delta_{n}\right\}$ where $\delta_{1}, \ldots, \delta_{n}$ are the degrees of $p$ in the respective variables $x_{1}, \ldots, x_{n}$ and $\left\{p_{k_{1}, \ldots, k_{n}} \in \mathbb{R},\left(k_{1}, \ldots, k_{n}\right) \in \Delta\right\}$ denotes its coefficient set. Alternatively, given multi-indices $I=\left(i_{1}, \ldots, i_{n}\right) \in \mathbb{N}^{n}$ and $\delta=\left(\delta_{1}, \ldots, \delta_{n}\right) \in \mathbb{N}^{n}$, the polynomial $p$ of degree $\delta$ can be written as follows:

$$
p(x)=\sum_{I \leq \delta} p_{I} x^{I} \text { with } p_{I} \in \mathbb{R}, \forall I \leq \delta,
$$

where $x^{I}=x_{1}^{i_{1}} \ldots x_{n}^{i_{n}}$ and $I \leq \delta$ is the order relation given by $i_{j} \leq \delta_{j}$ for all $j \in\{1, \ldots, n\}$.

\section{B. Multi-affine functions}

Multi-affine functions form a particular class of multivariate polynomials. Essentially, a multi-affine function is a function which is affine in each of its variables when the other variables are regarded as constant:

Definition 1: A multi-affine function $p: \mathbb{R}^{n} \rightarrow \mathbb{R}$ is a multivariate polynomial in the variables $x_{1}, \ldots, x_{n}$ where the degree of $p$ in each of the variable is at most 1 . For $x=\left(x_{1}, \ldots, x_{n}\right)$,

$$
p(x)=\sum_{\left(l_{1}, \ldots, l_{n}\right) \in\{0,1\}^{n}} p_{l_{1}, \ldots, l_{n}} x_{1}^{l_{1}} \ldots x_{n}^{l_{n}}
$$

where $p_{l_{1}, \ldots, l_{n}} \in \mathbb{R}$ for all $\left(l_{1}, \ldots, l_{n}\right) \in\{0,1\}^{n}$.

Belta and Habets prove [BH06] that a multi-affine function is uniquely determined by its values at the vertices of a rectangle.

Lemma 1: For all $x \in R_{n}, p(x)$ is a convex combination of the values at the set of vertices $V_{n}$.

We easily deduce that: $\min _{x \in R_{n}} p(x)=\min _{v \in V_{n}} p(v)$.

\section{Blossoming principle}

The blossoming principle provides a technique to prove bounds on multivariate polynomials over a compact set. The basic idea is to rewrite a polynomial as a multi-affine function and use well known bounds on multi-affine functions (see [Sei93] and references therein).

Let $p: \mathbb{R}^{n} \rightarrow \mathbb{R}$ be an arbitrary multivariate polynomial of degree $\delta:\left(\delta_{1}, \ldots, \delta_{n}\right)$ over variables $x_{1}, \ldots, x_{n}$, respectively. We convert $p(x)$ to a multi-affine function $q\left(z_{1}, \ldots, z_{n}\right)$, wherein each variable $x_{i}$ corresponds to multiple variables $z_{i, 1}, \ldots, z_{i, \delta_{i}}$ in the blossom.

Definition 2 (Blossom): The blossom or polar form of the polynomial $p: \mathbb{R}^{n} \rightarrow \mathbb{R}$ is the function $q_{\delta}: \mathbb{R}^{\delta_{1}+\cdots+\delta_{n}} \rightarrow \mathbb{R}$ given for $z=\left(z_{1,1}, \ldots, z_{1, \delta_{1}}, \ldots, z_{n, 1}, \ldots, z_{n, \delta_{n}}\right)$ by

$$
q_{\delta}(z)=\sum_{\left(l_{1}, \ldots, l_{n}\right) \in \Delta} p_{l_{1}, \ldots, l_{n}} \prod_{i=1}^{i=n} B_{l_{i}, \delta_{i}}\left(z_{i, 1}, \ldots, z_{i, \delta_{i}}\right)
$$

with

$$
B_{l, r}\left(z_{1}, \ldots, z_{r}\right)=\frac{1}{\left(\begin{array}{l}
r \\
l
\end{array}\right)} \sum_{\left\{\sigma_{1}, \ldots, \sigma_{l}\right\} \subseteq\{1, \ldots, r\}} z_{\sigma_{1}} \ldots z_{\sigma_{l}} .
$$

As an example, the blossom of the polynomial $p(x)=$ $3 x_{1}+2 x_{2}^{3}+x_{1}^{2} x_{2}^{2}$ of degree $\delta=(2,3)$ is

$$
\begin{aligned}
q_{\delta}(z)= & \frac{3}{2}\left(z_{1,1}+z_{1,2}\right)+2 z_{2,1} z_{2,2} z_{2,3} \\
& +\frac{1}{3} z_{1,1} z_{1,2}\left(z_{2,1} z_{2,2}+z_{2,1} z_{2,3}+z_{2,2} z_{2,3}\right) .
\end{aligned}
$$

Given $z=\left(z_{1,1}, \ldots, z_{1, \delta_{1}}, \ldots, z_{n, 1}, \ldots, z_{n, \delta_{n}}\right)$ and $z^{\prime}=$ $\left(z_{1,1}^{\prime}, \ldots, z_{1, \delta_{1}}^{\prime}, \ldots, z_{n, 1}^{\prime}, \ldots, z_{n, \delta_{n}}^{\prime}\right)$, we say $z \cong z^{\prime}$ iff, for all $k=1, \ldots, n$, there exists a permutation $\pi_{k}$ such that $\left(z_{k, 1}, \ldots, z_{k, \delta_{k}}\right)=\pi_{k}\left(z_{k, 1}^{\prime}, \ldots, z_{k, \delta_{k}}^{\prime}\right)$. It is easy to see that $\cong$ is an equivalence relation. The following proposition characterizes blossoms (equivalent to Definition 2):

Proposition 1: $q_{\delta}: \mathbb{R}^{\delta_{1}+\cdots+\delta_{n}} \rightarrow \mathbb{R}$ is a blossom of the polynomial $p: \mathbb{R}^{n} \rightarrow \mathbb{R}$ if and only if:

1) $q_{\delta}$ is a multi-affine function;

2) $q_{\delta}$ is a symmetric function of its arguments:

$$
\forall z \cong z^{\prime}, q_{\delta}(z)=q_{\delta}\left(z^{\prime}\right) ;
$$

3) $q_{\delta}$ satisfies the diagonal property:

$$
q_{\delta}\left(z_{1}, \ldots, z_{1}, \ldots, z_{n}, \ldots, z_{n}\right)=p\left(z_{1}, \ldots, z_{n}\right) .
$$

Having converted $p(x)$ to its blossom form $q_{\delta}(z)$, we employ the known bounds for a multi-affine polynomial over a rectangle (see Lemma 1). Given $x \in R_{n}=$ $\left[a_{1}, b_{1}\right] \times \cdots \times\left[a_{n}, b_{n}\right]$, we define the associated rectangle in the blossom as $R_{n}{ }^{\prime}=\prod_{k=1}^{k=n}\left[a_{k}, b_{k}\right]^{\delta_{k}}$ with vertices $V_{n}{ }^{\prime}=\prod_{k=1}^{k=n}\left\{a_{k}, b_{k}\right\}^{\delta_{k}}$. Then, it is easy to show that $\min _{x \in R_{n}} p(x) \geq \min _{v \in V_{n}^{\prime}} q_{\delta}(v)$.

The number of vertices $\left|V_{n}^{\prime}\right|$ in a blossom is exponential both in the degree and the number of variables in the polynomial $p$, making the application of Lemma 1 computationally expensive. We alleviate the exponential dependency on the number of variables by considering vertices equivalent under the permutation equivalence relation $\cong$.

Let ${\overline{V_{n}}}^{\prime}=\left(V_{n}^{\prime} / \cong\right)$ be the set of equivalence classes of the relation $\cong$ on the set $V_{n}^{\prime}$. It is easy to prove that $\left|{\overline{V_{n}}}^{\prime}\right|=\left(\delta_{1}+\right.$ $1) \times \cdots \times\left(\delta_{n}+1\right)$. From the second property in Proposition 1 , it follows that $\min _{x \in R_{n}} p(x) \geq \min _{\bar{v} \in \bar{V}_{n}^{\prime}} q_{\delta}(\bar{v})$.

Example 1: To illustrate this, let us consider a bivariate polynomial $p:[0,1] \times[2,3] \rightarrow \mathbb{R}$ with $\delta_{1}=1$ and $\delta_{2}=2$. Then $R_{2}^{\prime}=[0,1] \times[2,3]^{2}, V_{2}^{\prime}$ will contain $2^{3}=8$ vertices and ${\overline{V_{2}}}^{\prime}$ will contain $2 \times 3=6$ vertices shown below:

$$
\{\overline{(0,2,2)}, \overline{(1,2,2)}, \overline{(0,2,3)}, \overline{(1,2,3)}, \overline{(0,3,3)}, \overline{(1,3,3)}\} \text {. }
$$

Here, the bar over a tuple represents its equivalence class under the $\cong$ relation.

Thus, the blossoming principle can prove bounds for a multivariate polynomial $p(x)$ over a given rectangular set. We will now apply this principle to generate positive invariants.

\section{POLYHEDRAL INVARIANCE VERIFICATION BASED ON BLOSSOM ABSTRACTION}

In this section, we first consider the problem of proving positive invariance of a polyhedral set under polynomial dynamics. This naturally leads to a non-linear optimization problem, and the problem of showing that its solution is non-negative. We will attack this problem using a linear programming formulation that yields guaranteed lower bounds on the solution of the non-linear problem. We will consider the following dynamical system $S$ :

$$
\dot{x}(t)=f(x(t)), x(t) \in \mathbb{R}^{n}
$$


where $f: \mathbb{R}^{n} \rightarrow \mathbb{R}^{n}$ is a polynomial vector field written as a vector: $\left(f_{1}, \ldots, f_{n}\right)^{T}$ where each $f_{j}: \mathbb{R}^{n} \rightarrow \mathbb{R}$ is a polynomial. Let $\delta_{1, j}, \ldots, \delta_{n, j}$ be the respective degrees of the variables $x_{1}, \ldots, x_{n}$ for the polynomial $f_{j}$, and $\delta_{i}=$ $\max _{j \in\{1, \ldots, n\}} \delta_{i, j}$ for all $i \in\{1, \ldots, n\}$. The first step is to consider a "blossom form" of this system over a larger set of variables $z$, but with multi-affine dynamics in $z$.

\section{A. Blossom Form of Dynamical System}

For convenience, we "normalize" the degrees of all the polynomials $f_{i}$ in the vector field to $\delta:\left(\delta_{1}, \ldots, \delta_{n}\right)$ in the variables $x_{1}, \ldots, x_{n}$ respectively, by adding terms with zero coefficients wherever necessary. For all $i=1, \ldots, n$, let $f_{i, \delta}$ be the blossom for $f_{i}$ regarded as a polynomial of degree $\delta$ and let $f_{\delta}=\left(f_{1, \delta}, \ldots, f_{n, \delta}\right)$. Our abstract dynamical system $S^{\prime}$ will be of the form:

$$
\dot{z}(t)=q_{\delta}(z(t)), z(t) \in \mathbb{R}^{\delta_{1}+\cdots+\delta_{n}}
$$

where the abstraction vector field $q_{\delta}: \mathbb{R}^{\delta_{1}+\cdots+\delta_{n}} \rightarrow$ $\mathbb{R}^{\delta_{1}+\cdots+\delta_{n}}$ is given by: $q_{i, j, \delta}(z)=f_{i, \delta}(z)$ for all $i=1 \ldots, n$ and all $j=1, \ldots, \delta_{i}$ where we will denote by default $z=\left(z_{1,1}, \ldots, z_{1, \delta_{1}}, \ldots, z_{n, 1}, \ldots, z_{n, \delta_{1}}\right)$. We also need to define the vector space $H$ given by :

$$
H:\left\{z \in \mathbb{R}^{\delta_{1}+\cdots+\delta_{n}} \mid z_{j, 1}=\cdots=z_{j, \delta_{1}}, j=1, \ldots, n\right\} .
$$

The advantage of the abstraction is that the abstract system $\left(S^{\prime}\right)$ is multi-affine and we have the following lemma:

Lemma 2: The dynamical systems $S$ and $S^{\prime}$ are equivalent on the vector space $H$. If $x, z$ are trajectories of $S$ and $S^{\prime}$ such that $x_{i}(0)=z_{i, j}(0)$ for all $i=1, \ldots, n, j=1, \ldots, \delta_{i}$, and $z(0) \in H$ then $x_{i}(t)=z_{i, j}(t)$ for all $t \geq 0$.

Proof: It is easy to show that $H$ is an invariant of $S^{\prime}$. Therefore, $z(0) \in H$ implies that $z(t) \in H$ for all $t$. Next, using the diagonal property of the polar form we show that the systems (1) and (2) are equivalent if we restrict ourselves to the set $H$.

\section{B. Polyhedral Invariant Sets}

Let us fix a rectangle $R_{n}$ and a bounded polytope $P \subseteq R_{n}$ with a set of $m$ facets $\left\{F_{k} \mid k \in K=\{1, \ldots, m\}\right\}$ where

$F_{k}=\left\{x \in \mathbb{R}^{n} \mid a_{k} \cdot x=b_{k}\right.$, and $\left.a_{i} \cdot x \leq b_{i}, \forall i \in K \backslash\{k\}\right\}$,

it follows from the standard characterization of invariant sets (see e.g. [Aub91]) that $P$ is invariant for the dynamical system (1) if

$$
\forall k \in K, \forall x \in F_{k}, a_{k} \cdot f(x)<0
$$

where $a_{k}$ is the normal vector to $F_{k}$ pointing outside $P$. As pointed out in [ATS09] and by application of Tarski's Theorem [Tar48], this a decidable problem. However, the complexity of the decision procedure gives little hope for practical application. Let us remark that (3) can be reformulated as follows:

$$
\forall k \in K, \min _{x \in F_{k}}-a_{k} \cdot f(x)>0 .
$$

This consists in showing that the minimal values of the multivariate polynomials $-a_{k} \cdot f$ on the bounded polytopes $F_{k}$ are positive. Using the equivalence with the blossom form, this verification is equivalent to :

$$
\forall k \in K, \min _{z \in F_{k}^{\prime} \cap H}-a_{k}^{\prime} \cdot q_{\delta}(z)>0,
$$

wherein $a_{i}^{\prime}=\left(\frac{a_{i, 1}}{\delta_{1}}, . ., \frac{a_{i, 1}}{\delta_{1}}, . ., \frac{a_{i, n}}{\delta_{n}}, . ., \frac{a_{i, n}}{\delta_{n}}\right)$ and $F_{k}^{\prime}=\{z \in$ $\mathbb{R}^{\delta_{1}+\cdots+\delta_{n}} \mid a_{k}^{\prime} \cdot z=b_{k}$, and $\left.a_{i}^{\prime} \cdot z \leq b_{i}, \forall i \in K \backslash\{k\}\right\}$. Hence, if we are able to compute non-negative certified lower bounds of these minimal values, it is sufficient to prove that the polytope $P$ is invariant for the dynamical system (1). Therefore, the verification of polyhedral invariants for polynomial dynamical systems can be handled by solving a set of problems of optimization of multi-affine functions on bounded polytopes. More precisely, we have to deal with the following problem:

$$
\begin{array}{ll}
\text { minimize } & -a_{k}{ }^{\prime} \cdot q_{\delta}(z) \\
\text { over } & z \in R_{n}{ }^{\prime} \\
\text { subject to } & a_{i}{ }^{\prime} \cdot z \leq b_{i}, i \in K \backslash\{k\} \\
& a_{k}{ }^{\prime} \cdot z=b_{k}, \\
& z_{p, l}=z_{p, l+1}, p \in[1, n], l \in\left[1, \delta_{p}-1\right] .
\end{array}
$$

The principal result of $[\mathrm{BG} 12 \mathrm{a}]$ is a linear relaxation established by writing the Lagrangian of the previous problem and by using both blossom properties and the convexity property of multi-affine functions given by Lemma 1:

Theorem 1: The optimal value of problem (6) is greater than the optimal value $d_{k}{ }^{*}$ of:

$$
\begin{array}{lll}
\max & t & \\
& t \in \mathbb{R}, \lambda \in \mathbb{R}^{m_{I}}, \mu \in \mathbb{R}^{m_{J}}, & \\
\text { s.t. } & \lambda_{i} \geq 0, & i \in I, \\
& \left(\begin{array}{c}
t \quad \leq-a_{k}^{\prime} \cdot q_{\delta}(\bar{v}) \\
+\sum_{i \in K \backslash\{k\}} \lambda_{i}\left(a_{i}^{\prime} \cdot \bar{v}-b_{i}\right) \\
\end{array}\right. & k \in K \\
& +\mu\left(a_{k}^{\prime} \cdot \bar{v}-b_{k}\right) & \bar{v} \in \overline{V_{n}^{\prime}}
\end{array}
$$

where $\overline{V_{n}^{\prime}}=\left(V_{n}^{\prime} / \cong\right)$ is the quotient set of $V_{n}^{\prime}$ by the equivalence relation $\cong$.

If $d_{k}{ }^{*}$ are positive for all $k \in K$ then we can deduce that our polytope $P$ is invariant under $S$. We have then a sufficient condition for polyhedral invariant verification for polynomial dynamical systems.

We have presented the use of the blossom to check polyhedral invariants. Presently, we will now combine our ideas in an iterative scheme to generate polyhedral invariants.

\section{ITERATIVE APPROACH FOR THE COMPUTATION OF POLYHEDRAL INVARIANTS}

Having presented a scheme to check polyhedral invariance for polynomial dynamical systems, we will now present an iterative technique that attempts to generate such invariants. The $j^{\text {th }}$ iteration considers a candidate polyhedron $P_{j}$. Our approach will use the following steps: (a) Check if $P_{j}$ is invariant by computing the bounds $d_{k}^{*}$ given by the set of linear programs (7), each LP representing a face. If the obtained values of $d_{k}^{*}$ are all positive, we conclude that 
$P_{j}$ is a positive invariant set. (b) Otherwise, we modify the polyhedron $P_{j}$ using sensitivity analysis. Specifically, for each face $a_{i} x \leq b_{i}$ in $P_{j}$, we consider perturbations $\left(a_{i}+u_{i}\right) \cdot x \leq b_{i}+\alpha_{i}$ for perturbations $u_{i}$ and $\alpha_{i}$. We now consider the perturbation of the bound $p^{*}$ and $d^{*}$ from the problems (6) and (7).

\section{A. Gradient Descent}

The overall goal of our analysis is to find matrices $a_{1}, \ldots, a_{m}, b_{1}, \ldots, b_{m}$ written collectively as $A, b$, such that $\bigwedge_{k \in K} a_{k} x \leq b$ is a positive invariant of the system (1). Our approach can be viewed as a gradient ascent technique.

Consider the functional $\mathcal{F}(A, b)$ given by the optimal value $d^{*}$ obtained by solving problem (7) for given $A, b$. Our approach seeks to maximize this functional iteratively, stopping if we obtain $A, b$ that yield a positive $d^{*}$. We proceed as follows:

1) Start with $A_{0}, b_{0}$, our initial guess.

2) At each step, find "ascent directions" $U, \alpha$ such that $\mathcal{F}(A+U, b+\alpha)$ strictly improves the value of $\mathcal{F}$.

Since $\mathcal{F}$ is defined as the result of an optimization problem, finding the ascent direction is performed using sensitivity analysis.

\section{B. Sensitivity analysis}

An interesting feature of Lagrangian duality is that it enables sensitivity analysis (see e.g. [BV04]). Consider the following variation of problem (6):

$$
\begin{array}{lll}
\operatorname{minimize} & p(x) \\
\text { over } & x \in R, \\
\text { subject to } & \left(a_{i}+u_{i}\right) \cdot x \leq b_{i}+\alpha_{i}, \quad i \in I, \\
& \left(c_{j}+w_{j}\right) \cdot x=d_{j}+\beta_{j}, \quad j \in J,
\end{array}
$$

where $\alpha_{i} \in \mathbb{R}, u_{i} \in \mathbb{R}^{n}$ for all $i \in I$ and $\beta_{j} \in \mathbb{R}, v_{j} \in \mathbb{R}^{n}$ for all $j \in J$. This problem coincides with the original problem (6), when the vectors $\alpha=\left(\alpha_{i}\right)_{i \in I}, \beta=\left(\beta_{j}\right)_{j \in J}$ and the matrices $U=\left(u_{i}\right)_{i \in I}, W=\left(w_{j}\right)_{j \in J}$ are equal to zero. Therefore, if (6) is feasible then (8) is feasible, as well. Let $p^{*}$ and $p^{*}(U, W, \alpha, \beta)$ denote the optimal values of problems (6) and (8), respectively. Let $d^{*}$ and $d^{*}(U, W, \alpha, \beta)$ be the lower bounds of $p^{*}$ and $p^{*}(u, v, \alpha, \beta)$ obtained by application of Theorem 1. The following result shows how the solution of (7) allows us to compute a lower bound of $d^{*}(U, W, \alpha, \beta)$ and thus of $p^{*}(U, W, \alpha, \beta)$.

Theorem 2: Let $d^{*}$ and $\left(t^{*}, \lambda^{*}, \mu^{*}\right)$ be the optimal value and an optimal solution of the linear program (7). Then, for all $\alpha \in \mathbb{R}^{m_{I}}, U \in \mathbb{R}^{m_{I} \times n}$ and for all $\beta \in \mathbb{R}^{m_{J}}, W \in$ $\mathbb{R}^{m_{J} \times n}$ such that (8) is feasible we have:

$$
\begin{aligned}
& p^{*}(U, W, \alpha, \beta) \geq d^{*}(U, W, \alpha, \beta) \geq d^{*}-\lambda^{*} \cdot \alpha-\mu^{*} \cdot \beta \\
+ & \sum_{i \in I} \lambda_{i}^{*}\left(u_{i}^{\prime} \cdot \bar{v}\right)+\sum_{j \in J} \mu_{j}^{*}\left(w_{j}^{\prime} \cdot \bar{v}\right), \text { for all } \bar{v} \in \overline{V^{\prime}}
\end{aligned}
$$

Proof: By applying Theorem 1 to the perturbed problem (6), $d^{*}(U, W, \alpha, \beta)$ is the optimal value of

$$
\begin{array}{ll}
\operatorname{maximize} & t \\
\text { over } & t \in \mathbb{R}, \lambda \in \mathbb{R}^{m_{I}}, \mu \in \mathbb{R}^{m_{J}} \\
\text { subject to } & \lambda_{i} \geq 0, i \in I, \\
& t \leq q_{\delta}(\bar{v})+\sum_{i \in I} \lambda_{i}\left(\left[a_{i}^{\prime}+u_{i}^{\prime}\right] \cdot \bar{v}-b_{i}-\alpha_{i}\right) \\
& +\sum_{j \in J} \mu_{j}\left(\left[c_{j}^{\prime}+w_{j}^{\prime}\right] \cdot \bar{v}-d_{j}-\beta_{j}\right), \bar{v} \in \overline{V^{\prime}}
\end{array}
$$

The fact that $p^{*}(U, W, \alpha, \beta) \geq d^{*}(U, W, \alpha, \beta)$ is a consequence of Theorem 1. Let $\left(t^{*}, \lambda^{*}, \mu^{*}\right)$ be an optimal solution of the problem (7), let us show that $\left(t^{*}-\lambda^{*} \cdot \alpha-\mu^{*} \cdot \beta+\right.$ $\left.\sum_{i \in I} \lambda_{i}^{*}\left(u_{i}^{\prime} \cdot \bar{v}\right)+\sum_{j \in J} \mu_{j}^{*}\left(w_{j}^{\prime} \cdot \bar{v}\right), \lambda^{*}, \mu^{*}\right)$ is feasible for (9) for all $\bar{v} \in \overline{V^{\prime}}$. It is clear that $\lambda_{i}^{*} \geq 0$, for all $i \in I$. Also, for all $\bar{v} \in \overline{V^{\prime}}$

$$
\begin{aligned}
& \quad q_{\delta}(\bar{v})+\sum_{i \in I} \lambda_{i}^{*}\left(\left[a_{i}^{\prime}+u_{i}^{\prime}\right] \cdot \bar{v}-b_{i}-\alpha_{i}\right) \\
& +\sum_{j \in J} \mu_{j}^{*}\left(\left[c_{j}^{\prime}+u_{j}^{\prime}\right] \cdot \bar{v}-d_{j}-\beta_{j}\right) \\
& =q_{\delta}(\bar{v})+\sum_{i \in I} \lambda_{i}^{*}\left(a_{i}^{\prime} \cdot \bar{v}-b_{i}\right)+\sum_{j \in J} \mu_{j}^{*}\left(c_{j}^{\prime} \cdot \bar{v}-d_{j}\right) \\
& \quad-\lambda^{*} \cdot \alpha-\mu^{*} \cdot \beta+\sum_{i \in I} \lambda_{i}^{*}\left(u_{i}^{\prime} \cdot \bar{v}\right)+\sum_{j \in J} \mu_{j}^{*}\left(w_{j}^{\prime} \cdot \bar{v}\right), \\
& \geq t^{*}-\lambda^{*} \cdot \alpha-\mu^{*} \cdot \beta+\sum_{i \in I} \lambda_{i}^{*}\left(u_{i}^{\prime} \cdot \bar{v}\right)+\sum_{j \in J} \mu_{j}^{*}\left(w_{j}^{\prime} \cdot \bar{v}\right) . \\
& \text { Then, }\left(t^{*}-\lambda^{*} \cdot \alpha-\mu^{*} \cdot \beta, \lambda^{*}+\sum_{i \in I} \lambda_{i}^{*}\left(u_{i}^{\prime} \cdot \bar{v}\right)+\right. \\
& \left.\sum_{j \in J} \mu_{j}^{*}\left(w_{j}^{\prime} \cdot \bar{v}\right), \mu^{*}\right) \text { is feasible for }(9) \text { for all } \bar{v} \in \overline{V^{\prime}} \text {. It } \\
& \text { follows that } d^{*}(U, W, \alpha, \beta) \geq t^{*}-\lambda^{*} \cdot \alpha-\mu^{*} \cdot \beta+ \\
& \sum_{i \in I} \lambda_{i}^{*}\left(u_{i}^{\prime} \cdot \bar{v}\right)+\sum_{j \in J} \mu_{j}^{*}\left(w_{j}^{\prime} \cdot \bar{v}\right) \text { for all } \bar{v} \in \overline{V^{\prime}}, \text { which leads } \\
& \text { to the expected inequality since } d^{*}=t^{*} \text {. }
\end{aligned}
$$

\section{THE ITERATIVE APPROACH}

In this section, we are going to show how one constructs a polyhedral invariant for a given polynomial dynamical system $(S)$. The idea is to start from a given polyhedron, and use the previous sensitivity result to modify its matrix direction and its vector position in order to make it invariant. In fact, since the result given by Theorem 2 is linear on the perturbation variables, we will be able to construct a linear program trying to find suitable perturbations ensuring invariance of the perturbed polyhedra.

For this aim, let $U \in \mathbb{R}^{m_{K} \times n}$ (direction perturbation) and $\alpha \in R^{m_{K}}$, (position perturbation), and let $P_{U, \alpha}$ be the polytope given by

$$
P_{U, \alpha}=\left\{x \in \mathbb{R}^{n} \mid\left(a_{k}+u_{k}\right) \cdot x \leq b_{k}+\alpha_{k}, \forall k \in K\right\} .
$$

For $U=\alpha=0$, we recover the polytope $P$. We would like to find $\alpha$ and $U$ such that $P_{U, \alpha}$ is an invariant for (1). To establish the linear program, we should impose additional constraints on $P_{U, \alpha}$ in order to avoid degenerate cases: 
- $P_{U, \alpha} \subseteq R$.

- $P_{U, \alpha}$ is not empty.

- $P_{U, \alpha}$ is "relatively close" to $P$.

The first and the second constraints can be ensured by imposing bounds on the vector $b+\alpha: \underline{b} \leq b+\alpha \leq \bar{b}$. Here $\underline{b}$ is chosen to be a small but positive vector to trivially guarantee non-emptiness of $P_{U, j}$ by ensuring that $\overrightarrow{0} \in P_{U, j}$. However, the choice of $\bar{b}$ to guarantee that $P_{U, \alpha} \subseteq R$ is not clear. If $U$ were fixed then, one could compute components of $\bar{b}$ by solving $\max \left(a_{k}+u_{k}\right) x$ s.t. $x \in R$ to compute $\bar{b}_{k}$. However, $U_{k}$ is not fixed here. Therefore, rather than enforce $P_{U, \alpha} \subseteq R$ in the search for $U, \alpha$, we will allow our update procedure to generate a candidate $U, \alpha$ and then test a posteriori, whether our optimal choice respects the condition $P_{U, \alpha} \subseteq R$.

The third condition is ensured by imposing bounds $-\varepsilon_{\alpha} \leq$ $\alpha \leq \varepsilon_{\alpha},-\varepsilon_{U} \leq U \leq \varepsilon_{U}$ where $\varepsilon_{\alpha} \in \mathbb{R}^{m_{K}}$ and $\varepsilon_{U} \in$ $\mathbb{R}^{m_{K} \times n}$ are respectively parameter vector and matrix that can be tuned.

Now, denoting for $k \in K, d_{k}^{*}(U, \alpha)$ the optimal values of the relaxation of the perturbed problem for the polytope $P_{U, \alpha}$, the sensitivity analysis given in Theorem 2 gives us

$$
\forall k \in K, d_{k}^{*}(U, \alpha) \geq d_{k}^{*}-\lambda_{k}^{*} \cdot \alpha+\sum_{i=1}^{m} \lambda_{k}^{*}(i)\left(u_{i}^{\prime} \cdot \bar{v}\right) .
$$

where $d_{k}^{*}$ and $\left(t_{k}^{*}, \lambda_{k}^{*}\right)$ are the optimal values and solutions of problems (7) for polytope $P$ and $k \in K$ (where the equality constraint multiplier is the given by the $k$ component of $\lambda_{k}^{*}$ which will be denoted $\lambda_{k}^{*}(k)$ ). Then, for $P_{\alpha}$ to be an invariant polytope for the dynamical system (1), it is sufficient that for all $k \in K, d_{k}^{*}-\lambda_{k}^{*} \cdot \alpha+\sum_{i=1}^{n} \lambda_{k}^{*}(i)\left(u_{i}^{\prime} \cdot \bar{v}\right) \geq 0$. In order to find a suitable matrix perturbation $U$ and a suitable perturbation vector $\alpha$, we can solve the following problem:

$$
\begin{array}{ll}
\operatorname{maximize} & \min _{k \in K}\left(d_{k}^{*}-\lambda_{k}^{*} \cdot \alpha+\sum_{i=1}^{m} \lambda_{k}^{*}(i)\left(u_{i}^{\prime} \cdot \bar{v}\right)\right) \\
\text { over } & \alpha \in \mathbb{R}^{m_{K}}, U \in \mathbb{R}^{m_{K} \times n}, \bar{v} \in \overline{V^{\prime}} \\
\text { subject to } & \frac{\alpha_{i}}{u_{i, j}} \leq \alpha_{i} \leq u_{i, j} \leq \overline{\alpha_{i}}, i \in K \\
& \underline{u_{i, j}}, i \in K, j \in\{1, \ldots, n\}
\end{array}
$$

where $\underline{\alpha_{i}}=\max \left(-\varepsilon_{i}, \underline{b_{i}}-b_{i}\right), \overline{\alpha_{i}}=\min \left(\varepsilon_{i}, \overline{b_{i}}-b_{i}\right)$ and $u_{i, j}, \overline{u_{i, j}}$ are respectively the $j$-th component of the $i$-th row $\overline{\text { of }-} \varepsilon_{U}$ and $\varepsilon_{U}$. This problem can be recast as the following linear program:

$$
\begin{array}{ll}
\operatorname{maximize} & t \\
\text { over } & t \in \mathbb{R}, \alpha \in \mathbb{R}^{m_{K}}, U \in \mathbb{R}^{m_{K} \times n}, \\
\text { subject to } & t \leq d_{k}^{*}-\lambda_{k}{ }^{*} \cdot \alpha \\
& +\sum_{i=1}^{m} \lambda_{k}^{*}(i)\left(u_{i}^{\prime} \cdot \bar{v}\right), \bar{v} \in \overline{V^{\prime}} \\
& \frac{\alpha_{i} \leq \alpha_{i} \leq \overline{\alpha_{i}}, i \in K}{u_{i, j} \leq u_{i, j} \leq \overline{u_{i, j}}, i \in K, j \in\{1, \ldots, n\} .}
\end{array}
$$

If $\left(t^{*}, \alpha^{*}, U^{*}\right)$ is infeasible, we note that our algorithm fails to find a descent direction and must be restarted (by changing the bounds or the initial guess). Otherwise, let $\left(t^{*}, \alpha^{*}, U^{*}\right)$ be a solution of this linear program. If the optimal value of this problem is positive, then it is sufficient to prove that $P_{U^{*}, \alpha^{*}}$ is an invariant for the dynamical system (1). If the optimal value is strictly negative, then we compute $d_{k}^{*}\left(U^{*}, \alpha^{*}\right)$ by solving problems (7) for the polytope $P_{U^{*}, \alpha^{*}}$ for all $k \in K$. If all $d_{k}^{*}\left(U^{*}, \alpha^{*}\right)$ are nonnegative, then $P_{U^{*}, \alpha^{*}}$ is invariant. If the verification fails as well, then we use sensitivity analysis to modify $P_{U^{*}, \alpha^{*}}$ in order to find an invariant. This gives an iterative approach for synthesis of polytopic invariants of polynomial dynamical systems.

Remark 1: Let us remark that the polytope $P_{U^{*}, \alpha^{*}}$ computed by solving (10) may have empty facets. This results, for the empty facet $F_{k}$, in an unbounded value $d_{k}^{*}\left(U^{*}, \alpha^{*}\right)=$ $+\infty$. In order to avoid such situations, it may be useful to replace $\alpha^{*}$ by $\tilde{\alpha}^{*}$ such that $P_{U^{*}, \alpha^{*}}$ has no empty facet and $P_{U, \alpha^{*}}=P_{U^{*}, \tilde{\alpha}^{*}}$. Once again, this can be checked by solving a set of linear programs.

\section{NUMERICAL RESULTS}

We have implemented our approach in Matlab. In this section, we present some numerical results and compare results obtained with those from our previous work [BG12a]. All computations terminate within 10 seconds on a standard MACbook pro laptop.

1) Comparison experiments: We applied our approach to the FitzHugh-Nagumo system [Fit61] modeling the electrical activity of a neuron:

$\dot{x}_{1}=x_{1}-x_{1}^{3} / 3-x_{2}+\frac{7}{8}, \quad \dot{x}_{2}=0.08\left(x_{1}+0.7-0.8 x_{2}\right)$.

This system is known to have a limit cycle. Using our

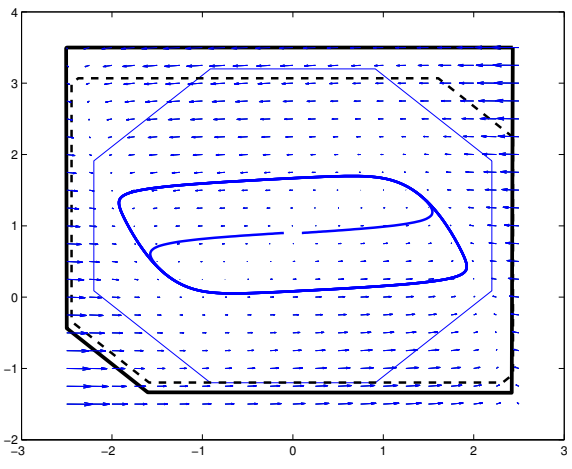

Fig. 1. Polyhedral invariants with and without matrix perturbations.

approach, we synthesized an invariant polytope containing the limit cycle. Working in the rectangle $[-2.5,2.5] \times$ $[-1.5,3.5]$, we found invariants polytope with 8 facets with uniformly distributed orientations (octagon). In Figure 1, we compare the invariant we found (represented with solid line) with the case where no matrix perturbation is applied (represented with dotted line). We can see that we obtain a bigger polyhedral invariant when the number of iteration is 
the same ( 8 iterations in that case). We now consider the Phytoplankton growth model [BG02]:

$$
\dot{x}_{1}=1-x_{1}-\frac{x_{1} x_{2}}{4}, \dot{x}_{2}=\left(2 x_{3}-1\right) x_{2}, \dot{x}_{3}=\frac{x_{1}}{4}-2 x_{3}^{2} \text {. }
$$

This system has a stable equilibrium. Using our approach, we synthesized an invariant polytope containing the equilibrium. Working in the rectangle $[0,3] \times[-0.1,2] \times[0,0.6]$ and using as candidate a a regular octagon with $m_{K}=18$ facets. Our previous approach needs 11 iterations to find the invariant polytope $\left(\varepsilon_{i}=0.1\right.$ for all $\left.i=1, \ldots, 18\right)$ depicted in the upper part of the figure. By adding direction freedom (the matrix perturbation bound $\varepsilon_{U}$ is taken such that all its values are equal to $\varepsilon=0.2$ ), we only need 6 iteration to find the invariant (see the down part of Figure 2).
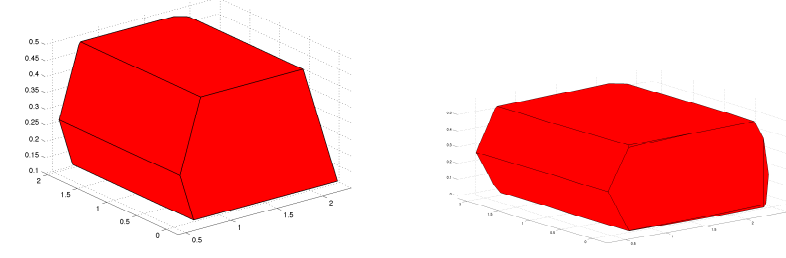

Fig. 2. Polytopic invariant in the left obtained after 11 iterations (previous approach) and the one in the left obtained after 6 iterations by adding direction freedom.

a) Dealing with Local Minima: Consider the well known Van Der Pol (VDP) reversed time oscillator [VV28]:

$$
\dot{x}_{1}=-x_{2}, \quad \dot{x}_{2}=-x_{2}\left(1-x_{1}^{2}\right)-x_{1} .
$$

This system is known to have a stable limit cycle. Since we reverse the time, the equilibrium become stable. If our working rectangle is $[-1,1]^{2}$ (not inside the limit cycle): starting with an octagon inside this rectangle, the approach will fail to find an invariant due to a local minimum. This means that we reach a iterate that does not have an ascent direction but also fails to be an invariant. To solve this problem, whenever a local minimum is reached, we replace our working rectangle by the bounding box of the reached polyhedra. By doing so, we allow our sensitivity result to find an ascent direction at the price of finding a smaller invariant (see Figure 3).

\section{CONCLUSIONS}

In this paper, an approach allowing the computation of polyhedral invariants for polynomial systems is proposed. Thanks to the additional degrees of freedom on the template direction and the use of bounding boxes, the given methods can be efficient in practice and the choice of the polyhedral guess could be made arbitrarily in many cases. A direct extension of this work will be to find the proper way to use bounding boxes (in order to get more precision) without getting smaller invariants. Also, one should get a smart way to find appropriate bounds that can ensure fast convergence without causing degeneracy. Finally, an important future work will be the extension of the approach to deal with more general non linear dynamical systems.

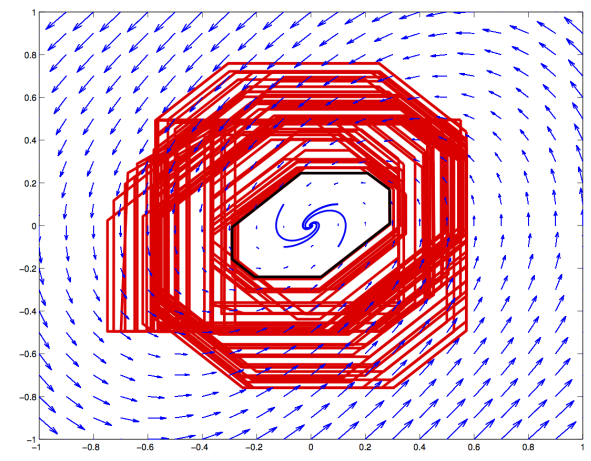

Fig. 3. The local minimum problem for the time reversed VDP.

Acknowledgments: Ben Sassi and Sankaranarayanan were supported by the US National Science Foundation (NSF) under CAREER Award \# 0953941. Girard was partially supported by the LabEx PERSYVAL-Lab (ANR-11-LABX0025-01). All opinions expressed are those of the authors and not necessarily of the NSF.

\section{REFERENCES}

[ATS09] A. Abate, A. Tiwari, and S. Sastry. Box invariance for biologically-inspired dynamical systems. Automatica, 45(7):16011610,2009

[Aub91] J.P. Aubin. Viability Theory. Birkhauser, 1991.

[BG02] O. Bernard and J.-L. Gouze. Global qualitative description of a class of nonlinear dynamical systems. Artificial Intelligence, 136:29$59,2002$.

[BG12a] M.A.Ben Sassi and A. Girard. Computation of polytopic invariants for polynomial dynamical systems using linear programming. Automatica, 2012.

[BG12b] M.A.Ben Sassi and A. Girard. Controller synthesis for robust invariance of polynomial dynamical systems using linear programming. Systems and Control Letters, 61(4):506-512, 2012.

[BH06] C. Belta and L.C.G.J.M. Habets. Controlling a class of nonlinear systems on rectangles. IEEE Transactions on Automatic Control, 51(11):1749-1759, 2006.

[BV04] S. Boyd and L. Vandenberghe. Convex Optimization. Cambridge University Press, 2004.

[Fit61] R. FitzHugh. Impulses and physiological states in theoretical models of nerve membrane. Biophysical J., 1:445-466, 1961.

[KHJ13] M. Korda, D. Henrion and C. N. Jones. Convex computation of the maximum controlled invariant set for polynomial control systems. Submitted, 2013.

[PJP07] S. Prajna, A. Jadbabaie, and G. J. Pappas. A framework for worstcase and stochastic safety verification using barrier certificates. IEEE Transactions on Automatic Control, 52(8):1415-1429, 2007.

[San10] S. Sankaranarayanan, Automatic invariant generation for hybrid systems using ideal fixed points. Hybrid Systems: Computation and Control, pp 221-230, 2010.

[Sei93] H.-P. Seidel. An introduction to polar forms. IEEE Comput. Graph. Appl., 13:38-46, 1993.

[Tar48] A. Tarski. A Decision Method for Elementary Algebra and Geometry. University of California Press, 1948.

[KKK95] M. Krstić, I. Kanellakopoulos and P. Kokotović. Nonlinear and Adaptive Control Design. Wiley Press, 1995.

[VV28] Balth. Van Der Pol and J. Van Der Marka, The heartbeat considered as a relaxation oscillation and an electrical model of the heart, The London, Edinburgh, and Dublin Philosophical Magazine and Journal of Science,vol. 6, no. 38, 1928. 